\title{
Determining Products of Inertia for Small Scale UAVs
}

\author{
Joseph S. Lorenzetti ${ }^{1}$ \\ Purdue University, West Lafayette, IN, 47906 \\ Leonel C. Bañuelos ${ }^{2}$ \\ Cal Poly San Luis Obispo, San Luis Obispo, CA, 93407 \\ Robert Clarke ${ }^{3}$, Oscar J. Murillo ${ }^{4}$, and Albion H. Bowers ${ }^{5}$ \\ NASA Armstrong Flight Research Center, Edwards, CA, 93523
}

\begin{abstract}
Moments of inertia and products of inertia often need to be determined for aircraft. As complex bodies, their mass properties need to be determined experimentally for best accuracy. While several moment of inertia experimental techniques have been developed, there are few to determine the products of inertia. Products of inertia can be easily determined mathematically if the angle between the aircraft $x$ body axis and principal $x$ axis is known. This method finds the principal inclination angle by mathematically correlating the measured moments of inertia about a range of axes of the aircraft. This correlation uses a least squares error minimization of a mathematical model that describes the ellipse of inertia in the aircraft's $x-z$ axes plane. Results from a test conducted on a small scale UAV at NASA Armstrong Flight Research Center is also presented, which is an example of the intended application of this technique.
\end{abstract}

\section{Nomenclature}

$I_{x} \quad=$ aircraft $\mathrm{x}$ body axis moment of inertia

$I_{y} \quad=$ aircraft $\mathrm{y}$ body axis moment of inertia

$I_{z} \quad=$ aircraft $\mathrm{z}$ body axis moment of inertia

$I_{x z} \quad=$ aircraft $\mathrm{x}-\mathrm{z}$ body axes product of inertia

$I_{\theta} \quad=$ moment of inertia about arbitrary axis in x-z plane

$\varphi \quad=$ angle between principal axes and arbitrary axes in $\mathrm{X}-\mathrm{Z}$ plane

$\theta \quad=$ angle between body axes and test point axes (pitch angle)

$\tau \quad=$ angle between principal axes and body axes

\section{Introduction}

$\mathrm{M}$ ASS properties, specifically moments and products of inertia, are important both in the design of aircraft and control systems for aircraft. These properties are constants that appear in the moment equations of motion for aircraft. Moments of inertia determination methods have been studied, and several experimental methods are able to provide accurate results. However, determining the products of inertia experimentally is more difficult.

Products of inertia can be thought of as describing the degree of symmetry of an object about a particular axis of rotation. Unlike moments of inertia, products of inertia for a body can be positive, negative, or zero. A zero product implies that the distribution of area is equal about the axis of rotation. A non-zero product means that a single moment that results in rotation about one axis will induce a moment about a perpendicular axis due to the asymmetrical movement of area. This is sometimes referred to as moment coupling or secondary oscillations. For an aircraft, the assumed symmetry about the x-z plane means that the products of inertia $I_{y z}$ and $I_{x y}$ are assumed zero. Also, $I_{x z}$ is zero in the reference frame of the principal axis system of the aircraft, but often the aircraft body

\footnotetext{
${ }^{1}$ Undergraduate Student, School of Aeronautics and Astronautics

${ }^{2}$ Undergraduate Student, School of Biomedical Engineering

${ }^{3}$ Aerospace Engineer, Controls \& Dynamics, PO Box 273, MS 4840D, Edwards, CA 93523-0273

${ }^{4}$ Aerospace Engineer, Office of Education, PO Box 273, MS AERO, Edwards, CA 93523-0273

${ }^{5}$ Chief Scientist, PO Box 273, MS 2011, Edwards, CA 93523-0273
} 
axis system has an angular offset from the principal axis system. This means that the product of inertia $I_{x z}$ will often have a non-zero value, and thus it needs to be determined.

Techniques to find the value of $I_{x z}$ rely on first finding the offset angle between the body and principal axes, and then using an analytical expression to solve for $I_{x z}$. However, finding the offset angle, $\tau$, is difficult to do accurately. NACA Report $467^{1}$ uses an analytical approach where the moments of inertia about the $\mathrm{x}$ and $\mathrm{z}$ body axes are measured, and then a third moment of inertia is measured about an arbitrary axis in the $\mathrm{x}-\mathrm{z}$ plane. The product of inertia is then calculated using the equation below, where $\theta$ is the angle between the $\mathrm{x}$ body axis and the third axis in the $\mathrm{x}-\mathrm{z}$ plane. This method is highly prone to measurement error, a slight error in the measured value of the moments of inertia can cause large errors in the calculated product of inertia value. Experimental methods for determining the moments of inertia are quite accurate, but not enough that this method could be considered accurate for determining $I_{x z}$.

$$
I_{x Z}=\frac{I_{x} \cos ^{2} \theta+I_{Z} \sin ^{2} \theta-I_{\theta}}{\sin 2 \theta}
$$

NASA TR R $-433^{2}$ presents another method to find the product of inertia $I_{x z}$. A single point suspension system is set up with a set of springs in a common plane to provide restraint in the yaw and roll directions. An excitation of the yaw mode creates an oscillation motion, and the angle of the spring plane is then adjusted until the coupled roll oscillation becomes zero. The angle of the spring plane can then be used to find the product of inertia. This method requires a complex setup and care must be taken to ensure the springs are calibrated and pretensioned correctly, that no secondary spring effects are introduced, and that no extraneous oscillatory damping is present. Also, the amount of mounting structure that is required could easily outweigh a small UAV. This could greatly affect the accuracy of the values.

This paper provides an improved method for determining the products of inertia and principal inclination angle for small scale UAVs. A lightweight test structure is preferred due to the relatively low weight of the UAVs. Although the test structure is tested separately to understand how much inertia it adds to the system, an overly heavy structure could increase the error of the experiment. In addition, some small scale UAVs are used for research purposes and the configurations of the aircraft are constantly changing. This means that the mass properties of the UAV may need to be measured multiple times. A simple rig that would not need constant modification would be ideal to minimize the amount of work required to retest the aircraft. This method can be leveraged to larger aircraft as long as a testing rig could be designed to properly gather the data required, as described later in this report.

Mathematically, the moment of inertia about any axis can be written in terms of moments of inertia in other axes, and an angle between them. This leads to the concept of the ellipsoid of inertia, which is visually a plot of the moments of inertia for any axis orientation for a body through a given point. The semi-major and semi-minor axes of the ellipsoid of inertia correspond to the principal axes of the body. For an aircraft, when the x-z plane is assumed to be an axis of symmetry, this ellipsoid can be simplified to an ellipse. Thus, for a given aircraft, if the ellipse that describes the relationship between moments of inertia about different axis in the x-z plane is known, the principal inclination angle can be easily determined. For the method described in this report, generating the ellipse that describes this relationship requires testing the aircraft at several pitch angles to gather moment of inertia data for different axes in the $x-z$ plane. Due to its simplicity and accuracy, the bifilar pendulum torsion method, as described by Jardin and Mueller ${ }^{3}$, for moment of inertia determination is used. The aircraft is tested at ten degree increments between $-80^{\circ}$ nose down pitch and $90^{\circ}$ nose up pitch. This provides data about every ten degree increment axis of the aircraft. Three tests were run at each pitch angle to ensure measurement precision. The test rig and setup will be described later in this paper. Once the data is collected, the general mathematical relationship that describes the relationship between moments of inertia about different axes is used to fit the data in a least squares sense. The fit equation can be used to determine the principal inclination angle, which can be used along with $I_{x}$ and $I_{z}$ to directly calculate the product of inertia $I_{x z}$.

The results section of this paper describes how this method was used to calculate the product of inertia $I_{x z}$ for a small scale UAV at NASA Armstrong Flight Research Center. The setup rig requirements are shown, and the calculation method and results are discussed.

\section{Determining the Principal Inclination Angle}

The aircraft body axes are defined using the conventional aircraft coordinate frame located at the center of gravity. A principal axes coordinate frame also goes through the center of gravity, and is defined as the set of axes where the inertia tensor is diagonal, or the products of inertia are all zero. The y body axis, pointing out of the right 
wing, is coincident with the y principal axis because of the aircraft's symmetry along the $\mathrm{x}-\mathrm{z}$ plane. This allows our analysis of the ellipsoid of inertia to be simplified to looking at the axes lying on the $\mathrm{x}-\mathrm{z}$ plane.

\section{A. Mathematical Representation of Moments of Inertia About Axes in the X-Z Plane}

Moments of inertia and the product of inertia about a set of axes, $\mathrm{x}$ and $\mathrm{z}$ aircraft body axes for example, coincident with the center of gravity can be expressed by the following integrals.

$$
\begin{aligned}
& I_{z}=\int x^{2} d m \\
& I_{x}=\int z^{2} d m
\end{aligned}
$$

Note that these equations are for a 2D shape and $m$ is a generic mass element. If the aircraft body axes are used as a reference and a new set of orthogonal axes, $\boldsymbol{z}^{\prime}$ and $\boldsymbol{x}^{\prime}$, are rotated about the center of gravity through an angle, $\boldsymbol{\varphi}$, then distances along the $\boldsymbol{x}^{\prime}$-axis from the origin have the following relationship with the reference axes.

$$
x^{\prime}=x \cos (\varphi)+z \sin (\varphi)
$$

The moment of inertia about the $z^{\prime}$ axis, $I_{z \prime}$, can be expressed in terms of the original axes and $\varphi$ as shown in Eq. (4).

$$
\begin{gathered}
I_{z \prime}=\int\left(x^{\prime}\right)^{2} d m \\
=\int(x \cos (\varphi)+z \sin (\varphi))^{2} d m \\
=\cos ^{2}(\varphi) \int x^{2} d m+2 \sin (\varphi) \cos (\varphi) \int x z d m+\sin ^{2}(\varphi) \int z^{2} d m \\
=I_{z} \cos ^{2}(\varphi)+2 I_{x z} \sin (\varphi) \cos (\varphi)+I_{x} \sin ^{2}(\varphi)
\end{gathered}
$$

The second term, $I_{x z}$, in the final expression is the product of inertia. If a mass element, $m$, has some distribution over an $\mathrm{x}-\mathrm{z}$ coordinate system, the following integral defines the product of inertia of $m$.

$$
I_{x z}=\int x z d m
$$

For the development of $I_{z \prime}$, if the reference set of axes are chosen to be principal axes of the body instead of the body axes, then the product of inertia is zero. The expression for $I_{z \prime}$ becomes:

$$
I_{z^{\prime}}=I_{z p} \cos ^{2}(\varphi)+I_{x p} \sin ^{2}(\varphi)
$$

The equation above then describes the value of the moment of inertia about any axis $z^{\prime}$ in terms of the principal moments of inertia and the angle between the principal axes and the axis in question. This equation was used to estimate the values of $I_{z p}$ and $I_{x p}$ for the aircraft. The orientation of the principal axes were not known a priori, so the angle, $\varphi$, could not be measured directly. To account for this, the angle of the axis being tested was measured with respect to the body axes orientation, and the Eq. (6) was modified to include an offset, $\tau$.

$$
I_{z^{\prime}}=I_{z p} \cos ^{2}(\theta-\tau)+I_{x p} \sin ^{2}(\theta-\tau)
$$

This offset, $\tau$, is the principal inclination angle, or the angle between the body axes and the principal axes. The angle, $\theta$, is the measured angle between the axis being tested and the body $\mathrm{x}$ axis.

\section{B. Modeling Test Data}

As stated earlier, Eq. (7) above models the relation between the moments of inertia about any axis for the aircraft. Modeling a particular aircraft's mass properties with this equation requires values of $I_{z \prime}$ to be known for 
different axes in the x-z plane. MATLAB ${ }^{4}$ was used to perform the curve fitting. Eq. (7) was used and $I_{z p}, I_{x p}$, and $\tau$ were unknown parameters. It is important to note that the pitch angle, $\theta$, is with respect to the $\mathrm{x}$ body axis. Values for the unknown parameters are estimated using a least squares error function minimization. The least squares error is simply

$$
\text { Error }=\sum\left(I_{z \prime}-\text { Measured Value }\right)^{2}
$$

The MATLAB ${ }^{4}$ optimization function FMINSEARCH was used to minimize the error function by varying the parameters $I_{z p}, I_{x p}$, and $\tau$. Initial estimates for $I_{z p}, I_{x p}$, and $\tau$ are needed to ensure accuracy of the FMINSEARCH function. $\tau=0, I_{z p}=I_{z}$, and $I_{x p}=I_{x}$ are acceptable initial estimates because for most aircraft the principal inclination angle is small and thus the principal and body inertias are similar. $I_{z}$ and $I_{x}$ are the moments of inertia about the $\mathrm{z}$ and $\mathrm{x}$ body axes, respectively. After using the MATLAB ${ }^{4}$ optimization analysis for the error function, the values of $I_{z p}, I_{x p}$, and $\tau$ are discovered, and Eq. (7) above can be written for the specific aircraft in question.

Note that in some testing cases, due to the design of the testing apparatus, it may not be possible for the $0^{\circ}$ pitch and $90^{\circ}$ pitch angles $\left(I_{x}\right.$ and $I_{z}$ respectively) to be tested exactly. In these situations, the parametrically fit Eq. (7) could be used to evaluate the moments of inertia values for the $\mathrm{x}$ and $\mathrm{z}$ body axes. Also note that in this sutation, the initial estimates used with FMINSEARCH would be the measurements closest to $0^{\circ}$ pitch and $90^{\circ}$ pitch. An example of this situation occurring would be if the testing apparatus held the aircraft at pitch increments such as -5 $\mathrm{deg}, 5 \mathrm{deg}, 15 \mathrm{deg}$, etc. This situation occurred during testing of the UAV at NASA Armstrong and will be discussed more later.

\section{Determination of Product of Inertia $\mathbf{I}_{\mathbf{x z}}$}

After the principal inclination angle, $\tau$, has been determined, a simple equation can be used to calculate the product of inertia. Once again start with the relationship between two pairs of orthogonal axes going through the center of gravity of an aircraft; where $x^{\prime}$ and $y^{\prime}$ are orthogonal axes that are rotated at an angle $\tau$ from $\mathrm{x}$ and $\mathrm{y}$.

$$
\begin{aligned}
& x^{\prime}=x \cos \tau+z \sin \tau \\
& z^{\prime}=z \cos \tau-x \sin \tau
\end{aligned}
$$

The moment of inertia about the $x^{\prime}$ axis, $I_{x \prime}$, can be written in terms of $\mathrm{x}$ and $\mathrm{y}$

$$
d I_{x^{\prime}}=z^{\prime 2} d m=(z \cos \tau-x \sin \tau)^{2} d m
$$

Integrating the equation above and substituting the mathematical definition of moment of inertia yields

$$
I_{x \prime}=I_{x} \cos ^{2} \tau+I_{z} \sin ^{2} \tau-2 I_{x z} \sin \tau \cos \tau
$$

and using trigonometric identities allows that equation to simply be rewritten as

$$
I_{x \prime}=\frac{I_{x}+I_{z}}{2}+\frac{I_{x}-I_{z}}{2} \cos 2 \tau-I_{x z} \sin 2 \tau
$$

This equation is now a representation of the moment of inertia about an arbitrary axis, written as a function of the moments of inertia about two other orthogonal axes, their product of inertia, and the angle between the different coordinate frames. The product of inertia in the equation above is the product of inertia in the body coordinate frame that we are interested in.

$$
\frac{d I_{x \prime}}{d \tau}=-2\left(\frac{I_{x}-I_{z}}{2}\right) \sin 2 \tau-2 I_{x z} \cos 2 \tau=0
$$

Taking the derivative and setting the equation equal to zero allows us to now have an equation where $\tau$ is the principal inclination angle. This occurs because the manipulation of the equation finds the max value of $I_{x \prime}$, which is along one of the principal axes. 


$$
\tan 2 \tau=\frac{-2 I_{x z}}{I_{x}-I_{z}}
$$

Rearrangement yields Eq. (11) above. This equation allows us to directly calculate the product of inertia $I_{x z}$ in the body coordinate system by only knowing $I_{x}$ and $I_{z}$ in the body coordinate system, and the principal inclination angle $\tau$. The values of $I_{x}$ and $I_{z}$ are measured experimentally as mentioned before. In the special case when the experimental setup does not measure these values specifically, they can be found from Eq. (7) after it is fit to the collected data. Note that in this derivation, the angle, $\tau$, is the angle from the body axes to the principal axes, and not the other way around. If done incorrectly, the sign of the product of inertia will be different.

\section{NASA Armstrong PRANDTL-D Aircraft Example}

The technique for determining the products of inertia for a small scale UAV was used at NASA Armstrong on the PRANDTL-2 flying wing aircraft. The aircraft is part of the PRANDTL-D project which is investigating the aerodynamic characteristics of the bell shaped lift distribution and its potential application in commercial aviation. Processing data from the experimental test flights requires the mass properties of the aircraft to be known, including the products of inertia.

As mentioned earlier in this report, small scale UAV type aircraft are most suited to using this technique. This method requires moments of inertia to be calculated about many different axes of the test object, which could potentially be difficult for larger objects due to testing apparatus constraints. However, this method could theoretically be used with any solid body.

Determining the moments of inertia for a complicated geometric body requires experimental testing methods. The method used at NASA Armstrong for the PRANDTL-D aircraft is the commonly used bifilar pendulum method. Additionally, a specialized procedure presented by Jardin and Mueller ${ }^{3}$ was used for greater accuracy. This method includes a higher fidelity dynamic model of the testing device than previous methods. It also accounts for aerodynamic drag, viscous damping of the motion, and is based on a nonlinear model. A MATLAB ${ }^{4}$ Simulink model is used to perform a parameter estimation technique to match the nonlinear model to the observed oscillation data, thus generating the moment of inertia values.

\section{A. Bifilar Pendulum Testing Apparatus}

The testing apparatus for the bifilar pendulum test was specially designed to enable the moment of inertia values to be tested at a range of pitch angles. Below is a picture of the testing apparatus holding the PRANDTL-D flying wing aircraft, and the aircraft during flight tests.
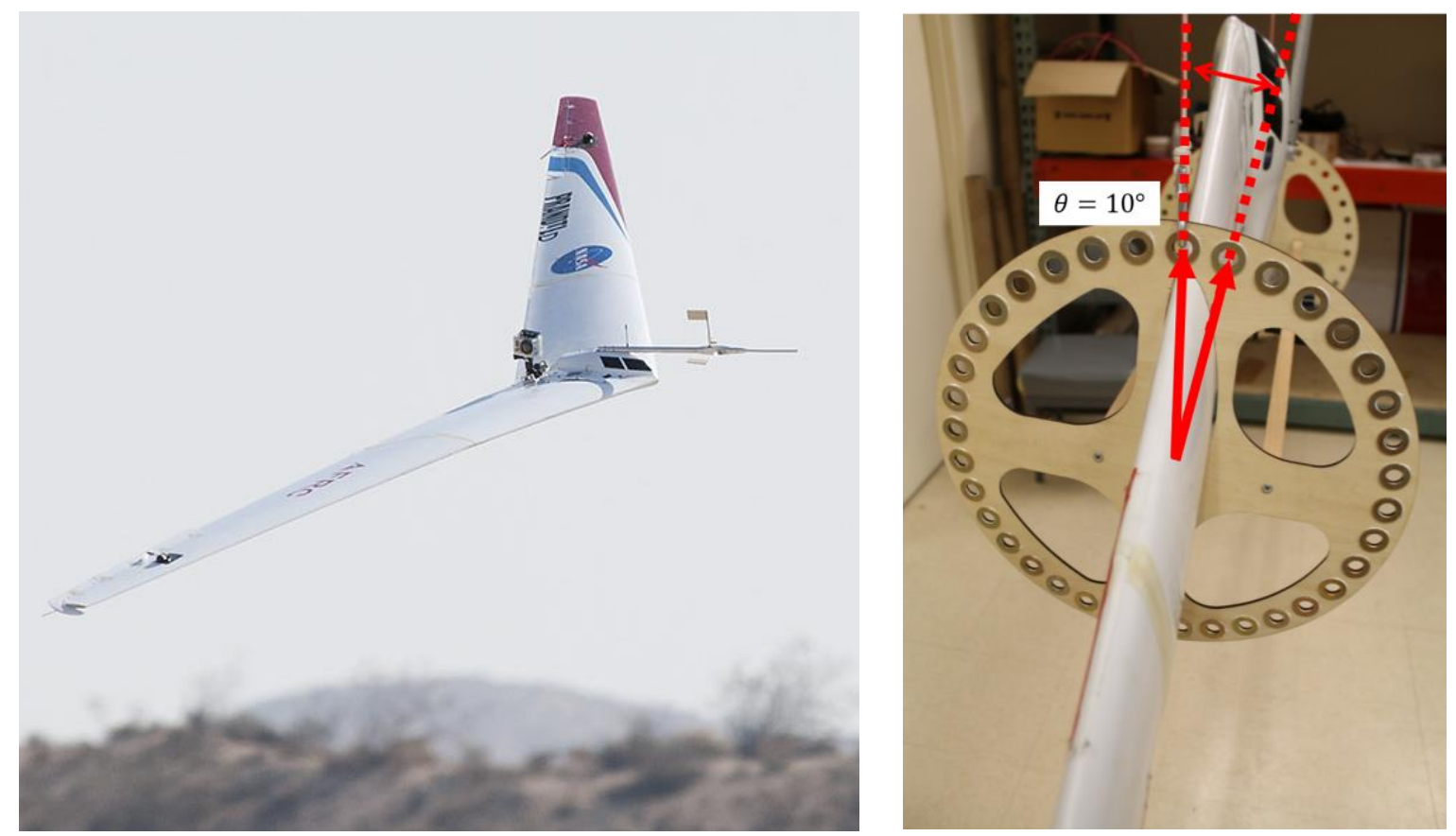

Figure 1. NASA Armstrong PRANDTL-D Aircraft (left) and Bifilar Pendulum Test Rig (right) 
As shown in the picture above, the testing apparatus consists of two circular plywood pieces. An airfoil shaped cut in the middle of the circular pieces allows them to slide over the sides of the aircraft wing to hold it in a fixed orientation. The holes on the outside of the disks are spaced at ten degree increments and reinforced with metal washers modified to have a knife edge inner surface. For the bifilar pendulum setup, the aircraft is hung by metal $\mathrm{S}$ hooks modified to have knife edges at the contact point with the disk's washers.

The aircraft sits so the center of gravity is located between the filars, and the pivot point where the assembly is hung allows the aircraft center of gravity to fall within the plane of the filars. This ensures that you are testing the moment of inertia about an axis that runs through the center of gravity, which is critical to getting accurate results. However, this also means that each tested pitch position may not be exactly ten degrees different, even though the mounting holes are spaced by ten degrees. Due to this side effect, it is important to measure the true $\mathrm{x}$ body axis angle (pitch angle) for each test and not just assume the pitch angle from the test apparatus design. Although this is inconvenient, it does not have an adverse effect on the results.

The most important aspect of the apparatus is that it enabled us to test for moments of inertia about many different axes. A wireless inertial measurement unit was attached to the rig to measure the angular rate data that was required for the post processing described earlier. The pendulum swinging motion was initiated by hand and at small disturbance angles. IMU data was streamed live to a laptop, and runs with unacceptable amounts of noise or secondary motions were stopped. The system was then allowed to settle before continuing with a new test run. Some coupling was expected in the motion about different axes, so unacceptable secondary motions were considered to be oscillations or motion with magnitudes that were above reasonable.

\section{B. Bifilar Pendulum Test Results}

Using the method presented by Jardin and Mueller $^{3}$ the aircraft was tested at approximately ten degree increments for a total of 18 different pitch angles. The results of the experiment are shown in the figure below.

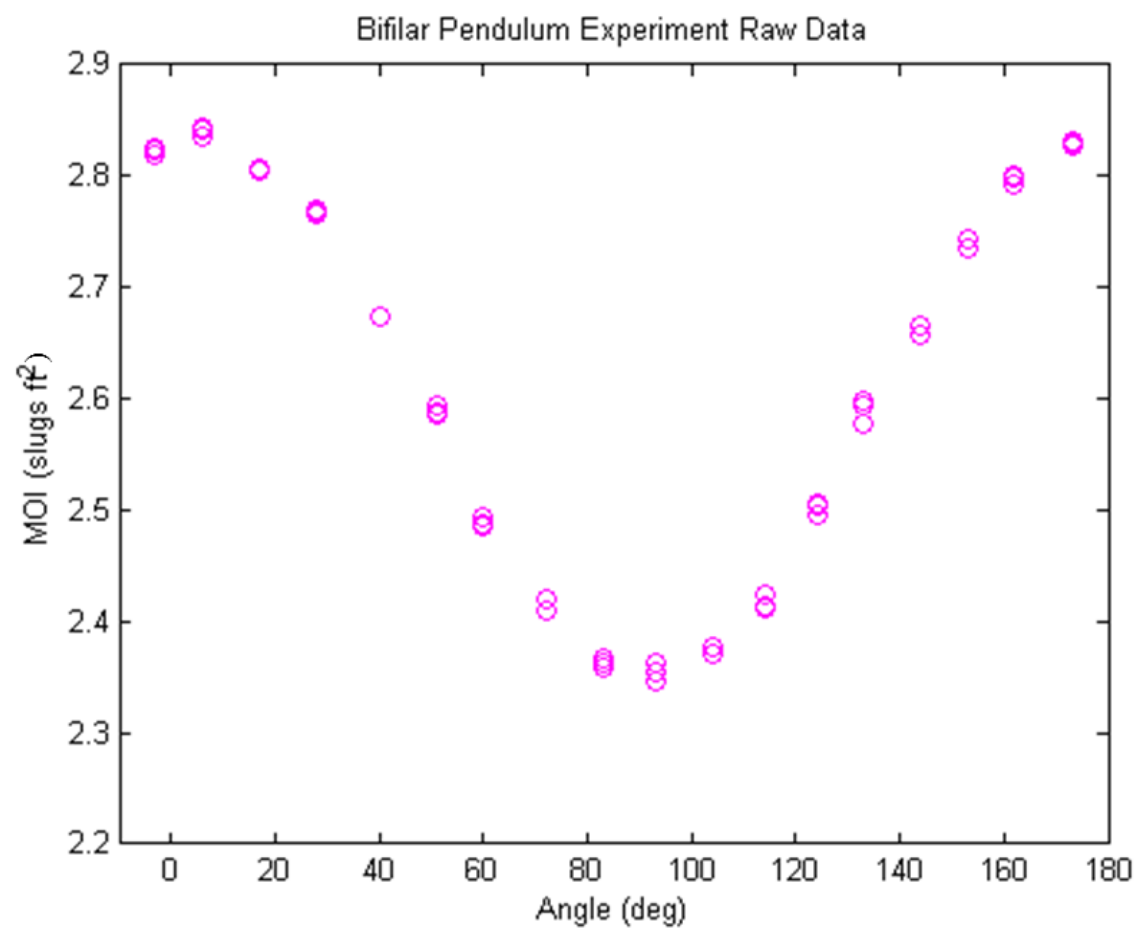

Figure 2. PRANDTL-D Bifilar Pendulum Moments of Inertia

Three trials were run at each pitch angle. Each trial was post processed with the MATLAB ${ }^{4}$ Simulink model discussed earlier to find the moment of inertia. All three calculated moment of inertia values at each pitch angle are plotted in Fig. 2. Note that the values presented above are the entire test setup moment of inertia minus the test rig moment of inertia. As mentioned, the test setup system consisted of both the aircraft and the rig. The test rig inertias were tested separately and were subtracted for the test setup system values in order to isolate the aircraft's properties. A standard deviation error analysis of the values at each angle is presented later in this report. 


\section{Determination of Product of Inertia from PRANDTL-D Data}

Using the FMINSEARCH function in MATLAB ${ }^{4}$, the least squares error, Eq. (8), was minimized, where $I_{z \prime}$, was given in Eq. (7). $I_{z p}, I_{x p}$, and $\tau$ were parameters that were varied in the FMINSEARCH function, and the angle $\theta$ was the angle collected from the experimental data. The predicted parameters were then used to plot an estimation of the moment of inertia and the errors were calculated.

As can be seen in the results shown in Fig. 3 below, the principal inclination angle was approximated as $\tau=2.1^{\circ}$. The data points are the same as in Fig. 2, and the blue dashed line is the least squares fit of Eq. (7). The red dotted line shows the location of the principal axis with respect to the body axes. The moments of inertia about the body axes are also shown in the figure.

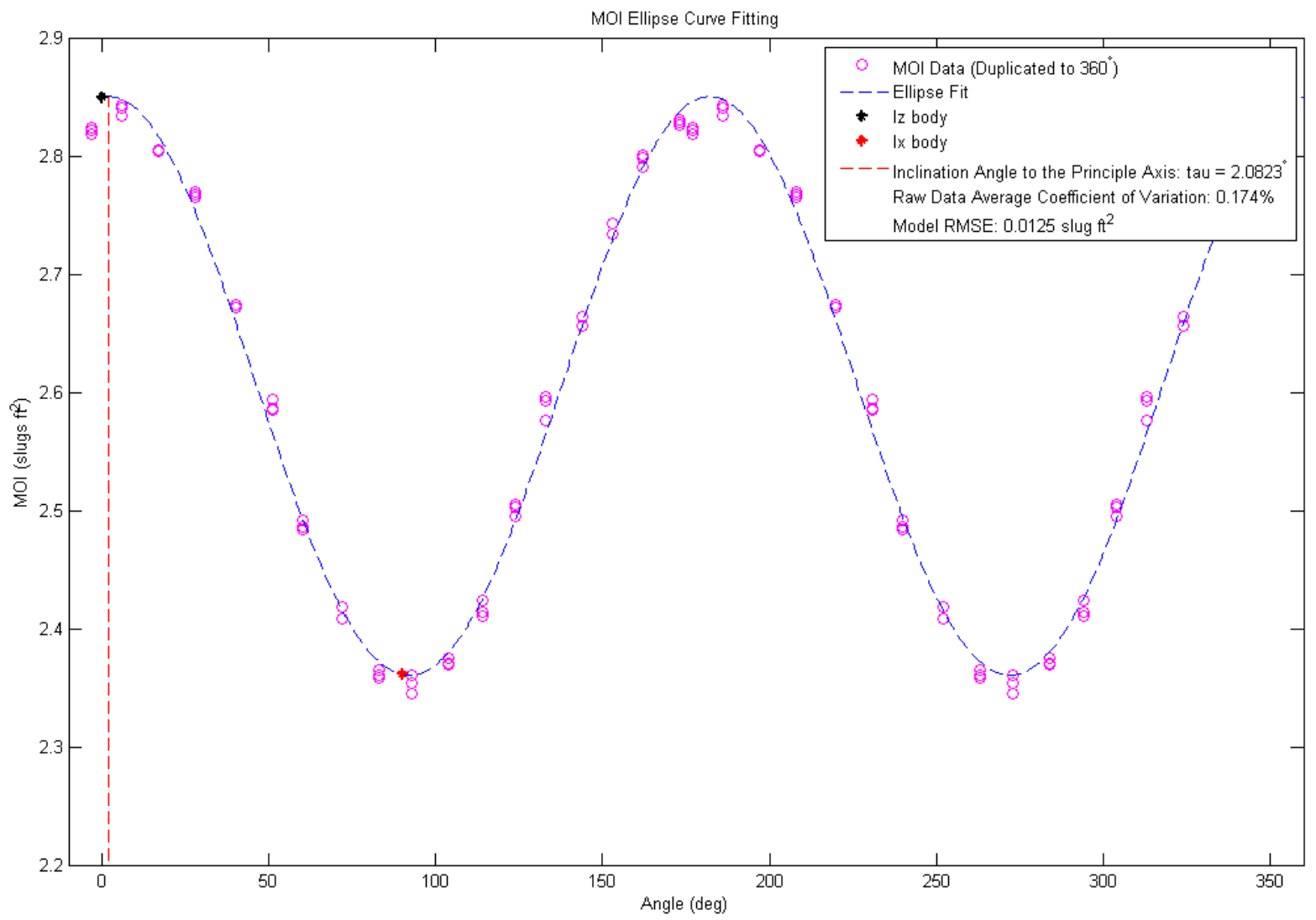

Figure 3. PRANDTL-D Processed MOI Data, Principal Inclination Angle Determination

Two errors were calculated for this example: average coefficient of variation of the raw data and root mean square error for the model least squares regression.

Table 1. PRANDTL-D Error Analysis

\begin{tabular}{|c|c|c|}
\cline { 2 - 3 } \multicolumn{1}{c|}{} & $\sigma$ & Coefficient of Variation \\
\hline Raw Data & 0.0045 & $0.1744 \%$ \\
\cline { 2 - 3 } & RSME [slugs- $\mathrm{ft}^{2}$ ] \\
\hline Linear Regression & 0.0125 \\
\hline
\end{tabular}

The standard deviation for the raw test data is the average of all standard deviations between test points at each angle. For the raw data, the coefficient of variation shows that one standard deviation is quite a bit less than $1 \%$ of 
the mean, which demonstrates the high precision and repeatability of the experiment. The root mean square error for the linear regression curve shows that the fit line is also less than $1 \%$ of the average moment of inertia value. This suggests that the fit of the predicted curve is very close to the test data.

For visual purposes, the moments of inertia and estimation results are also plotted in polar coordinates to demonstrate the elliptical shape of the moment of inertia-axis position relationship.

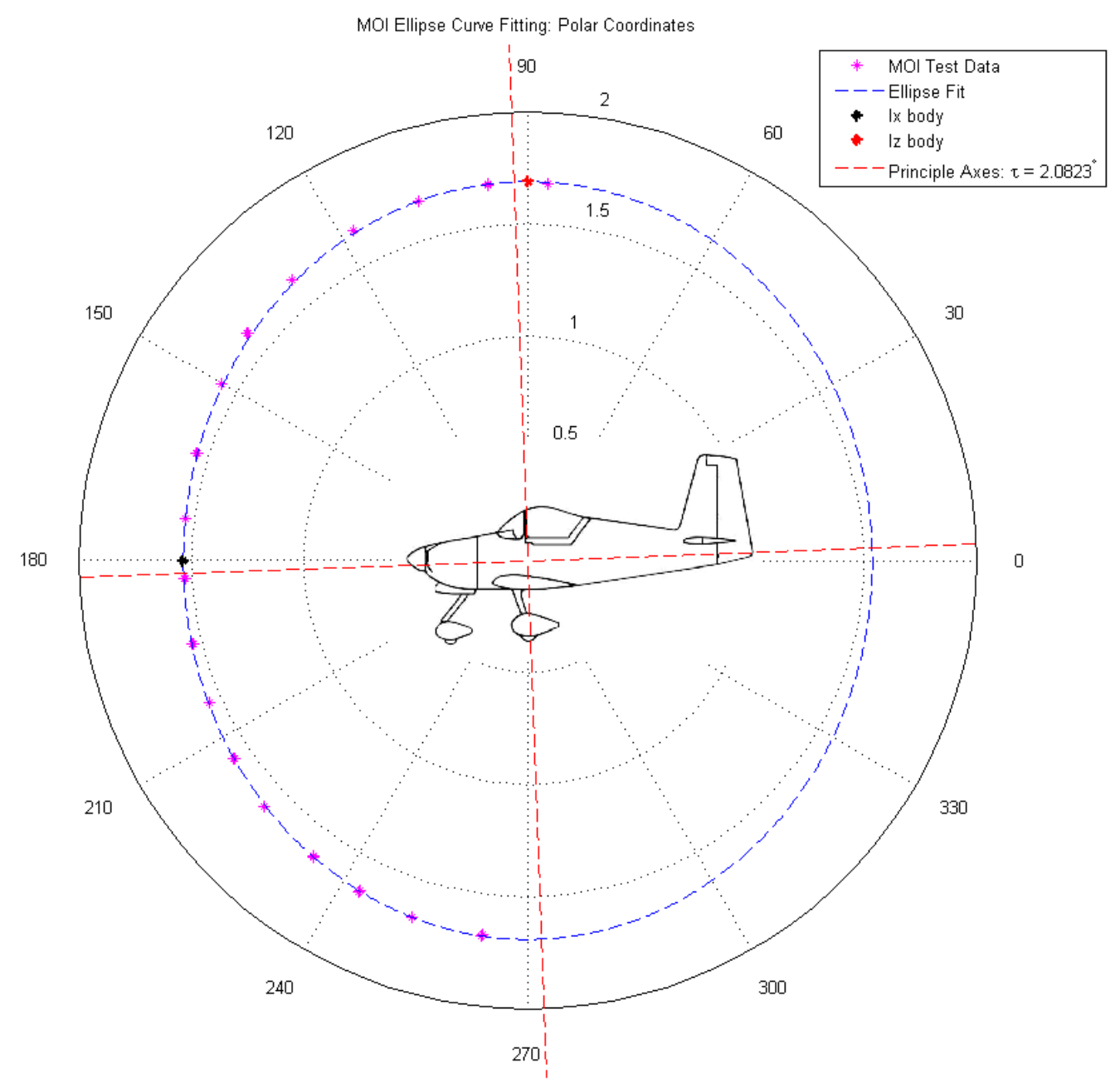

Figure 4. Ellipse of Inertia for PRANDTL-D Aircraft

Note that the red dotted axes are still the principal axes of the body. As can be seen, the principal axes are oriented such that if the aircraft was pitched nose up from horizontal by the angle $\tau$, then the principal x axis would be parallel to horizontal. This is shown graphically in the figure below.

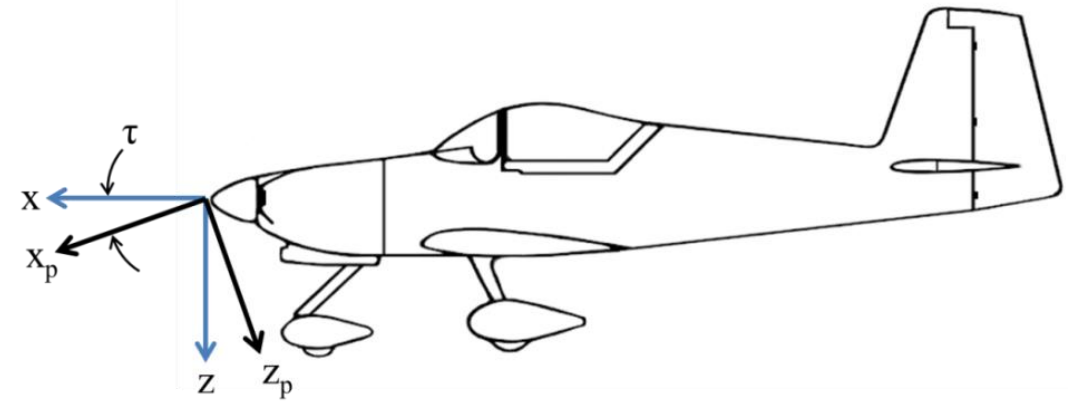

Figure 5. Principal and Body Axes Orientation

American Institute of Aeronautics and Astronautics 
Now that the principal inclination angle, $\tau$, and the $\mathrm{x}$ and $\mathrm{z}$ body axis moments of inertia are known, the product of inertia can be calculated using Eq. (11). As discussed earlier, the principal inclination angle value used in Eq. (11) is the angle from the body axes to the principal axes. In this case, $\tau$ is positive, based on the coordinate frame convention (as shown in the figure above). $I_{x z}$ was calculated for the PRANDTL-D aircraft and the result is shown in Table 2 below.

Table 2. PRANDTL-D Product of Inertia

\begin{tabular}{|c|c|}
\hline$I_{x}$ & 2.3596 slugs $\mathrm{ft}^{2}$ \\
\hline$I_{z}$ & 2.8477 slugs $\mathrm{ft}^{2}$ \\
\hline$\tau$ & $2.0875^{\circ}$ \\
\hline$I_{\boldsymbol{x} \boldsymbol{z}}$ & $\mathbf{0 . 0 1 7 8}$ slugs $\mathbf{f t}^{2}$ \\
\hline
\end{tabular}

\section{Conclusion}

Mass properties of aircraft are important characteristics to know. There are several developed techniques for experimentally determining the moments of inertia of aircraft, but not as many methods for determining the products of inertia. Current methods include calculating the product of inertia by knowing the moments of inertia about two orthogonal axes and a third angle in the same plane, and the angle between the third angle and the other axes. This method requires very precise measurements to be accurate. Another method uses a single point suspension test rig with a spring plane that opposes yaw and roll motion. This method is complex and requires a lot of setup and consideration. This new method provides a way to reliably determine the product of inertia experimentally while requiring only a simple experiment to be conducted. The main limitation of this method is that the test rig must be constructed to allow the aircraft to be mounted at different pitch angles, which could be difficult for some vehicles. For example it could limit the method's ability to be applied to large or heavy bodies. However, this technique is well suited for small scale UAV research aircraft.

\section{Appendix}

An alternate derivation of the equations that relate the moments of inertia about different axes to the angles between them (Eq. (6)) is shown here. This method also includes an alternate variation of the equation that calculates the product of inertia based on the principal inclination angle and the body axes moments of inertia (Eq. (11)).

The equation for the ellipse of inertia for the aircraft, with the center at the center of mass, has the form:

$$
I_{x} x^{2}+I_{z} z^{2}+2 I_{x z} x z=1
$$

Assuming that the ellipse is aligned with the principal axes of the aircraft, this equation can be simplified because the product of inertia is zero.

$$
I_{x p} x_{p}^{2}+I_{z p} z_{p}^{2}=1
$$

Moment of inertia values along any axis in the xz plane can be found by considering a simple coordinate frame transformation. In the figures below, it can be seen graphically that the values of the moment of inertia can be found about any axis by employing the rotation to the equation of the standard aircraft ellipse about the principal axes.

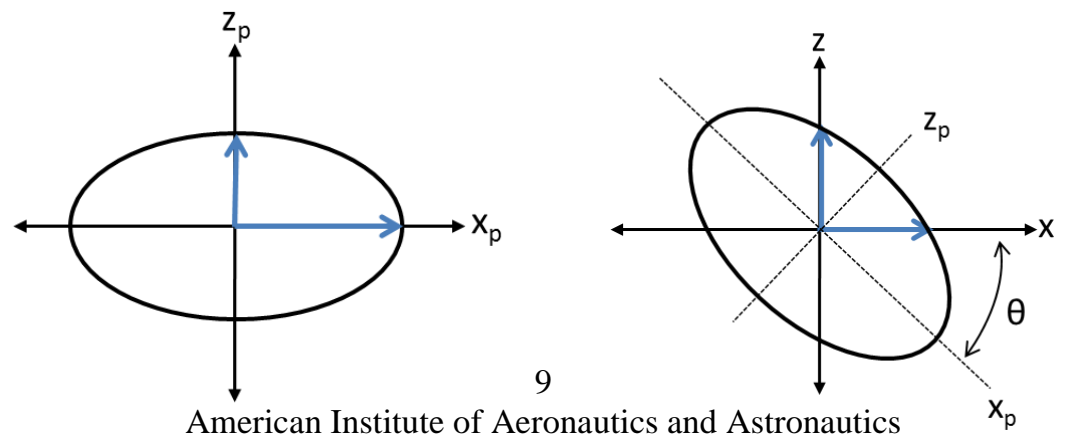




$$
\begin{aligned}
& x_{p}=x \cos \theta-z \sin \theta \\
& z_{p}=z \cos \theta+x \sin \theta
\end{aligned}
$$

These values are then substituted into Eq. (13) above and rearranged.

$$
\left(I_{x p} \cos ^{2} \theta+I_{z p} \sin ^{2} \theta\right) x^{2}+\left(I_{x p} \sin ^{2} \theta+I_{z p} \cos ^{2} \theta\right) z^{2}+2 \sin \theta \cos \theta\left(I_{z p}-I_{x p}\right) x z=1
$$

Notice that this equation is of the same form as Eq. (12), for an ellipse of inertia of the aircraft about any set of axes. The coefficients can be equated to give:

$$
\begin{aligned}
& I_{x}=I_{x p} \cos ^{2} \theta+I_{z p} \sin ^{2} \theta \\
& I_{z}=I_{x p} \sin ^{2} \theta+I_{z p} \cos ^{2} \theta
\end{aligned}
$$

These are the equations derived earlier, Eq. (6), where the moment of inertia about any axis can be found given the principal moments of inertia and the angle between the axes. Furthermore, an equation for product of inertia can be found. Note that now $\theta$ is actually $\tau$, the principal inclination angle. Once again equating the coefficients between Eq. (14) and Eq. (12) gives

$$
I_{x z}=\sin \tau \cos \tau\left(I_{z p}-I_{x p}\right)
$$

Multiplying both sides by $\left(\cos ^{2} \tau-\sin ^{2} \tau\right)$ gives

$$
\left(\cos ^{2} \tau-\sin ^{2} \tau\right) I_{x z}=\sin \tau \cos \tau\left(I_{z p}-I_{x p}\right)\left(\cos ^{2} \tau-\sin ^{2} \tau\right)
$$

Then distributing values on the right hand side and using trigonometric identities

$$
2(\cos 2 \tau) I_{x z}=\sin 2 \tau\left(I_{z p} \cos ^{2} \tau+I_{x p} \sin ^{2} \tau-I_{x p} \cos ^{2} \tau-I_{z p} \sin ^{2} \tau\right)
$$

Algebraically rearrange, and then use another trigonometric identity as well as substituting Eq. $(15,16)$

$$
\begin{gathered}
\frac{2 I_{x z}}{\left(I_{z p} \cos ^{2} \tau+I_{x p} \sin ^{2} \tau\right)-\left(I_{x p} \cos ^{2} \tau+I_{z p} \sin ^{2} \tau\right)}=\frac{\sin 2 \tau}{\cos 2 \tau} \\
\frac{-2 I_{x z}}{I_{x}-I_{z}}=\tan 2 \tau
\end{gathered}
$$

Eq. (17) is identical to Eq. (11). 


\section{Acknowledgments}

The authors wish to thank Robert "Red" Jensen and Derek Abramson from NASA Armstrong Flight Research Center. They designed and built the testing rig to hold the PRANDTL-D aircraft, and also provided guidance on testing procedures. Also, the authors would like to thank the NASA Armstrong PRANDTL Project intern team, especially Jack Toth, Cynthia Farr, and Heather Laffoon, for their help with the moment of inertia testing.

\section{References}

${ }^{1}$ Soulé, H. A., and Miller, M. P., "The Experimental Determination of the Moments of Inertia of Airplanes,” NACA R-467, 1934.

${ }^{2}$ Wolowicz, C.H., and Yancey, R.B., "Experimental Determination of Airplane Mass and Inertial Characteristics", NASA TR-433, Flight Research Center, Edwards, CA, Oct. 1974.

${ }^{3}$ Jardin, M. R., and Mueller, E. R., "Optimized Measurements of UAV Mass Moment of Inertia with a Bifilar Pendulum," AIAA-2007-6822, AIAA GNC Conference, Hilton Head, SC, Aug. 20-23, 2007.

${ }^{4}$ MATLAB, The MathWorks, Inc., Natick, Massachusetts, United States.

${ }^{5}$ Beer, F. P., Johnston, R., Comwell, P. J., and Mazurek, D. F., Vector Mechanics for Engineers: Statics, $10^{\text {th }}$ ed., The McGraw-Hill Companies, 2013.

${ }^{6}$ Marghitu, D. B., “Chapter 4: Moments of Inertia," Auburn MECH2110, URL:

http://www.eng.auburn.edu/ marghitu/MECH2110/C 4.pdf [cited 19 October 2014]. 